\title{
HEvAS: Headline Evaluation and Analysis System
}

\author{
Marina Litvak, Natalia Vanetik, Itzhak Eretz Kdosha \\ Software Engineering Department, \\ Shamoon College of Engineering, \\ Beer Sheva, Israel \\ \{marinal, natalyav, itzhaer\}eac.sce.ac.il
}

\begin{abstract}
Automatic headline generation is a subtask of one-line summarization with many reported applications. Evaluation of systems generating headlines is a very challenging and undeveloped area. We introduce the Headline Evaluation and Analysis System (HEvAS) that performs automatic evaluation of systems in terms of a quality of the generated headlines. HEvAS provides two types of metricsone which measures the informativeness of a headline, and another that measures its readability. The results of evaluation can be compared to the results of baseline methods which are implemented in HEvAS. The system also performs the statistical analysis of the evaluation results and provides different visualization charts. This paper describes all evaluation metrics, baselines, analysis, and architecture, utilized by our system.
\end{abstract}

\section{Introduction}

A headline of a document can be defined as a short sentence that gives a reader a general idea about the main contents of the story it entitles. There have been many reported practical applications for headline generation (Colmenares et al., 2015) or related tasks. Automatic evaluation of automatically generated headlines is a highly important task, where a candidate headline is assessed with respect to (1) readability (i.e. whether the headline is easy to understand), and (2) relevance (i.e. whether the headline reflects the main topic of an article). Building unbiased metrics that manage to make objective evaluations of these properties has been proved to be a difficult task. Some of the related work resort to human-assisted evalua- tion (Zajic et al., 2002), which is undoubtedly expensive and time-consuming. Therefore, most of works rely on the existing tools for automatic evaluation such as ROUGE (Shen et al., 2016; Hayashi and Yanagimoto, 2018). The main assumption being that because the metrics work well for standard summaries, the same applicable to short summaries and headlines, as a private case. However, authors of (Colmenares et al., 2015) provide statistical evidence that this statement does not necessarily hold. We suspect that the main reason is that a summary needs to convey the content of a document while a headline should introduce, but not describe, the main subject of a document. Moreover, even very short summaries usually include at least two full sentences while headlines do not. Despite that discovery, not many attempts to develop special metrics for the headline evaluation were made. Two new metrics - an adaptation of a ROUGE metric, and a metric for comparing headlines on a conceptual level using Latent Semantic Indexing (LSI) — were introduced in (Colmenares et al., 2015).

\section{Related Work}

This section surveys the metrics used recently in literature for a headline evaluation task and approaches we use for the introduced metrics as part of HEvAS. For the rest of this paper the terms "reference headline" and "candidate headline" will be used to address the human-generated and the automatically generated headlines, respectively.

\subsection{ROUGE metrics}

ROUGE metrics (Lin, 2004) are widely used for evaluation of summaries, aiming to identify content overlap — in terms of word n-grams - between gold-standard (reference) summaries and the evaluated (system) summary.

\section{ROUGE-N}


This recall-oriented metric measures the number of $N$-grams in the reference headline that are also present in a candidate headline. It is defined as: $\frac{\mid n \text {-grams }(R) \cap n \text {-grams }(C) \mid}{\mid n \text {-grams }(R) \mid}$, where $R$ refers to the reference headline, $C$ to the candidate headline, and the function $n$-grams returns the set of contiguous $N$-grams of words in a text. In our system we use the ROUGE-N metric with $N=1$ and $N=2$. ROUGE-SU

One of the problems of using the ROUGE-N metric (with $N>1$ ) is that requesting headlines to share contiguous $N$-grams might be a very strong condition. This is even more problematic when taking into account that headlines are comprised, on average, of 8-10 tokens. This metric combines ROUGE-1 with a relaxed version of ROUGE-2 that takes into account non-contiguous (skip) bigrams. For example, "President Trump said" will produce three skip bigrams: "President Trump," "President said," and "Trump said." Let's denote a function that returns all unigrams of the headline $H$ as $1-\operatorname{grams}(H)$, and a function that returns its skip-bigrams as $s 2$-grams $(H)$. Then formally, $R O U G E-S U(R, C)$ is defined as follows: $\frac{|s u(R) \cap s u(C)|}{|s u(R)|}$, where $s u(H)=1-\operatorname{grams}(H)$ s2-grams $(H)$. By allowing gaps between bigrams, this metric detects similarities among phrases that differ by adjectives, or small changes.

\section{ROUGE-WSU}

The main problem of ROUGE-SU is that it gives the same importance to all skip-bigrams extracted from a phrase. For instance, suppose that the following phrases were compared: $H_{1}$ : " $x B C x x$ ", $H_{2}$ : "B yyyC", $H_{3}$ : " $z z B z C$ ". The only skip-bigram they all have in common is " $B$ $C$,", and ROUGE-SU gives us the same similarity score between the three of them. Authors of (Colmenares et al., 2015) proposed to weight the skip-bigrams with respect to their average skip-distance. Formally, it must be calcu-

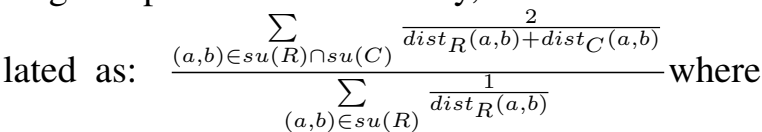
function $\operatorname{dist}_{H}(a, b)$ returns the skip distance between words " $a$ " and " $b$ " in headline $H$. For unigrams, the function returns 1 . This measure produces different scores for $\mathrm{H}_{2}$ and $\mathrm{H}_{3}$ in our example. Namely, ROUGE-WSU $\left(H_{1}, H_{3}\right)>$ ROUGE$\operatorname{WSU}\left(H_{1}, H_{2}\right)$.

\subsection{Averaged Kullback-Leibler divergence}

The Kullback-Leibler divergence is a measure of how two probability distributions are different. It is widely used for measuring the similarity between texts, as the distance between the probability distributions of their words. However, the KL-divergence is not symmetric and cannot be used as a distance metric. Therefore, the averaged KL-divergence is used instead, which is defined as follows (Huang, 2008): $D_{\operatorname{AvgKL} L}\left(\overrightarrow{t_{a}} \| \overrightarrow{t_{b}}\right)=$ $\sum_{t=1}^{m}\left(\pi_{1} \times D\left(w_{t, a} \| w_{t}\right)+\pi_{2} \times D\left(w_{t, b} \| w_{t}\right)\right)$, where $\overrightarrow{t_{a}}$ is a vector representation of a text (document or headline in our case) $a, w_{t, a}$ is a weight ${ }^{1}$ of term $t$ in a text $a, \pi_{1}=\frac{w_{t, a}}{w_{t, a}+w_{t, b}}, \pi_{2}=$ $\frac{w_{t, b}}{w_{t, a}+w_{t, b}}$, and $w_{t}=\pi_{1} \times w_{t, a}+\pi_{2} \times w_{t, b}$.

\subsection{Latent Semantic Indexing}

The ROUGE and KL-Divergence metrics relate two headlines only on the basis of word cooccurrences, i.e., they compare headlines at a very low syntactic level (token matching). We also need other metrics that are able to detect abstract concepts in the text and useful for both comparing headlines at a semantic level and measuring of a headline's coverage of a document topics. For this end, authors of (Colmenares et al., 2015) decided to use Latent Semantic Indexing (LSI) to extract latent concepts from a corpus and represent documents as vectors in this abstract space. The similarity was then computed by means of angular distances. The exact steps that were performed in (Colmenares et al., 2015), are as follows: (1) a document-TF-IDF matrix $M$ is built; (2) Singular Value Decomposition (SVD) is performed on $M$ resulting in matrices $U S V^{T}$; (3) the eigenvalues in matrix $S$ are analyzed and filtered; (4) the transformation matrix $V S^{-1}$ is calculated, which enables the translation of TF-IDF document vectors to vectors in latent space; (5) after computing latent space vectors for both the headline and the entire document, their cosine similarity is calculated.

\subsection{Topic Modeling}

Topic model is a type of statistical model for discovering the abstract "topics" that occur in a collection of documents. Latent Dirichlet allocation (LDA) (Blei et al., 2003; Blei, 2012) allows documents to have a mixture of topics. LDA uses a

\footnotetext{
${ }^{1}$ The $t f-i d f$ (term frequency inverse document frequency) weighting scheme or a basic $t f$ (term frequency) can be used.
} 
generative probabilistic approach for discovering the abstract topics, (i.e., clusters of semantically coherent documents). In particular, we define a word as the basic discrete unit of any arbitrary text, which can be represented as an item $w$ indexed by a vocabulary $\{1,2, \cdots,|V|\}$. A document is then a sequence of $N$ words denoted by $\mathbf{w}=\left(w_{1}, w_{2}, \cdots, w_{N}\right)$. Finally, we define a corpus of $M$ documents as $\mathbf{D}=\left\{\mathbf{w}_{1}, \mathbf{w}_{2}, \cdots, \mathbf{w}_{M}\right\}$. LDA finds a probabilistic model of a corpus that not only assigns high probability to members of the corpus, but also assigns high probability to other similar documents (Blei et al., 2003).

\subsection{Word Embeddings}

Word embeddings is another approach for building a semantically-enriched text representation, which provides a good basis for comparison between two texts at the semantic level. Word embeddings represent words as dense high-dimension vectors. These dense vectors model semantic similarity, i.e., semantically similar words should be represented by similar dense representations while words with no semantic similarity should have different vectors. Typically, vectors are compared using a metric such as cosine similarity, euclidean distance, or the earth movers distance (Kusner et al., 2015). Two well-known methods to acquire such dense vector representations are word2vec (Mikolov et al., 2013) and GloVe (Pennington et al., 2014). Both methods are based on the concept of distributional semantics, which exploits the assumption that similar words should occur in similar surrounding context.

\subsection{Readability Assessment}

Generation of readable headlines is not an easy task. Therefore, evaluation of headlines must include readability measurements. Most works in this area are based on a key observation that vocabulary used in a text mainly determines its readability. It is hypothesized that the use of commonfrequently occurring in a language-words makes texts easier to understand. Because it was observed that frequent words are usually short, word length was used to approximate the readability instead of frequency in many works (Kincaid et al., 1975; Gunning, 1952; Mc Laughlin, 1969; Coleman and Liau, 1975). According to (DuBay, 2004), more than 200 formulae for measuring readability exist. A survey of readability assessment methods can be found in (Collins-
Thompson, 2014). However, most of readability metrics are designed for larger texts and not applicable for a single headline.

\section{The HEvAS System}

HEvaS aims at evaluation of systems for headline generation in terms of multiple metrics, both from informativeness and readability perspectives. The results can be analyzed and visualized. This section describes all metrics and system settings that can be specified by the end user.

\subsection{Informativeness Metrics in HEvAS}

In this paper, we propose 12 informativeness metrics for headline evaluation, some are novel and some are adopted from the literature, which comprise the base for the introduced evaluation framework.

\section{ROUGE metrics}

ROUGE-1,2,SU, and WSU metrics are used for measuring similarity between a candidate and reference headlines.

\section{Averaged KL-Divergence}

We used averaged KL-Divergence for measuring both (1) similarity between the generated headline and its reference title, and (2) the headline's coverage of important keywords representing a document, as its similarity to the document.

\section{TM-based metrics}

We apply LDA topic modeling ${ }^{2}$ on the input documents. The following outputs of the LDA algorithm, normalized and treated as probabilities, are relevant to our studies: (1) Topic versus word dictionary, which gives the word $w$ distributions $P\left(w \mid P_{i}\right)$ for each topic $P_{i}$; (2) Inferred topic distributions for each document $d$ in the studied corpus, namely the probability $P\left(P_{i} \mid d\right)\left(\theta_{i}\right.$ parameter of the LDA model) that a certain document $d$ belongs to a topic $P_{i}$; (3) Importance of every topic in a document $d, P\left(d \mid P_{i}\right)$.

Given the LDA's output, we compute vector representations in a topics space for headlines (candidate and reference) and their documents, as follows: Each headline $H$ and each document $d$ are represented by a vector over $K$ topics, where each topic $P_{i}$ is assigned a weight computed as a normalized sum of word-in-document-topic importance $P\left(w \mid P_{i}\right) P\left(P_{i} \mid d\right) P\left(d \mid P_{i}\right)$ over all words $w$ in $P_{i}$. In order to evaluate a headline, two metrics are calculated: (a) the headline's coverage of im-

\footnotetext{
${ }^{2}$ Mallet tool (Graham et al., 2012) was used.
} 
portant topics representing a document, as a cosine similarity between the headline and the document vectors; and (b) similarity to the reference headlines, as a cosine similarity between the headline and the references vectors.

\section{LSI-based metrics}

We adopt the LSI-DS metric from (Colmenares et al., 2015) for measuring a headline's coverage of latent topics of its document. In addition, we extend it to the similarity between system and reference headlines by computing latent space vectors for both types of headlines and measuring a cosine similarity between their vectors. Also, our system allows a user to decide how to filter (if at all) the number of eigenvalues: by absolute number, by ratio, or by filtering out the values below a specified threshold.

\section{Word Embedding-based metrics}

This metric is based on Google's word2vec model, in which every word from the English vocabulary is assigned with a 300-dimension vector. We use the average vector (as a standard) to represent multiple words. For example, a headline is represented by an average vector calculated from representations of all its words. Similarity between two representations is measured by cosine similarity, which may imply similarity in content. As such, also two types of metrics are supported: (1) the headline's coverage of important topics representing a document, as a cosine similarity between the headline and the document vectors; (2) similarity to the reference headlines.

\subsection{Readability Metrics in HEvAS}

Currently, HEvAS contains the following five metrics: (1) Proper noun ratio (PNR). It is hypothesized that higher PNR indicates higher readability (Smith et al., 2012), because proper nouns contribute to a text disambiguation. (2) Noun ratio (NR). NR is used to capture the proportion of nouns present in the text. The text with lower proportion of nouns is considered to be easier to read (Hancke et al., 2012). (3) Pronoun ratio (PR). PR is a linguistic measure indicating the level of semantic ambiguity that can arise while searching for the concept that a pronoun represents.(Štajner et al., 2012) A text with lower PR is considered more readable. (4) Gunning fog index. In linguistics, the Gunning fog index (Gunning, 1952) is a readability test for English writing. We use the following formula: $F o g=0.4 *$ $\left(\#\right.$ words $\left.+8 * \frac{\# \text { complex words }}{\# \text { words }}\right)$, where \#words is the headline length. (5) Average word length (AWL). The AWL reflects the ratio of long words used in a text. It was proven that the use of long words makes a text more difficult to understand for dyslexics. (Rello et al., 2013)

\subsection{Baselines}

For comparative evaluations and a possibility to get impression about relative performance of the evaluated systems, five baselines are implemented in HEvAS: (1) First compiles a headline from nine first words; (2) Random extracts nine first words from a random sentence; (3) $\boldsymbol{T F}-\boldsymbol{I D F}$ selects nine top-rated words ranked by their $t f-i d f$ scores; (4) WTextRank generates a headline from nine words extracted by the TextRank algorithm (Mihalcea and Tarau, 2004) for the keyword extraction; (5) STextRank extracts nine first words from the top-ranked sentence by the TextRank approach for extractive summarization.

\subsection{Statistical analysis and visualization}

To determine whether the difference between system scores is statistically significant, the statistical significance test must be applied. HEvaS performs Tukey test (Jones and Tukey, 2000) if the results are normally distributed, and Wilcoxon test (Bergmann et al., 2000) otherwise.

To visualize the results of evaluation, the system generates the following plots for all evaluated systems and chosen metrics: (1) Bar plot (with or without confidence intervals); (2) Box plot (five number summary); (3) Scatter graph for visualizing cross-correlation between metrics.

\subsection{HEvAS Implementation}

The system is implemented in Java as a standalone application and is available for download ${ }^{3}$ in a .zip archive ${ }^{4}$. The demo video is provided. ${ }^{5}$ HEvAS provides the following options to the end user: (1) Provide input files. The documents, their gold titles, and the generated headlines must be provided as an input for every evaluation run. The documents with their (reference) headlines must be provided as one (xml-like formatted) file;

\footnotetext{
${ }^{3}$ The current version of HEvAS supports only Windows OS.

${ }^{4}$ https://drive.google.com/file/d/ 1-7Z--XMfmlbzjzyKlFOLfCKDEvAm0eNq/view? usp=sharing

${ }^{5}$ https://drive.google.com/open?id= 1BoaV9CUoZHJqMfAC1peRXsSCka 4-3jeO
} 
and all headlines generated by one system are also must be organized in one file. ${ }^{6}$ All files are required to be UTF-8 plain texts in English. (2) Specify output files. All results, including the summarizing statistics and charts (specified by the user), are saved to the file system. The folder for those files location must be provided by the user. (3) Choose metrics. The user can specify which category (informativeness or readability) of metrics and which metrics from each category she wants to apply in the evaluation process. Some metrics are also must be configured with additional settings. For example, LSI metrics require additional settings for optional filtering latent eigenvalues; (4) Choose charts. The user can specify which charts she wants to use for the visualization of the evaluation results. (5) Choose baselines. The user may specify which baselines to use for the comparative evaluations. Figure 1 depicts the flowchart of the HEvAS system, with its main modules.

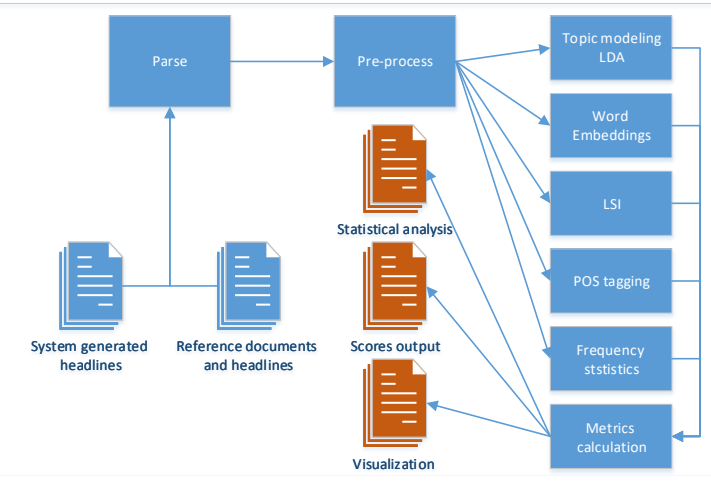

Figure 1: HEvAS data flow

Once evaluation is finished, its results are visualized at the system's interface and written to the file system. For every headline generation system the output file (in csv format) is generated, where columns stand for chosen metrics and rows stand for the input documents. Also, one summarizing csv file is generated where all systems can be ranked by their avg metric scores. One single score for each system is calculated as an average for every metric. Additionally, an average score over all metrics is calculated for every system; this is possible because all of the metrics are $[0,1]$-normalized. Figure 2 shows an example of

\footnotetext{
${ }^{6}$ The examples of such files are provided with the soft-
}

final average scores of competing systems as generated by HEvAS. Figure 3 shows an example of

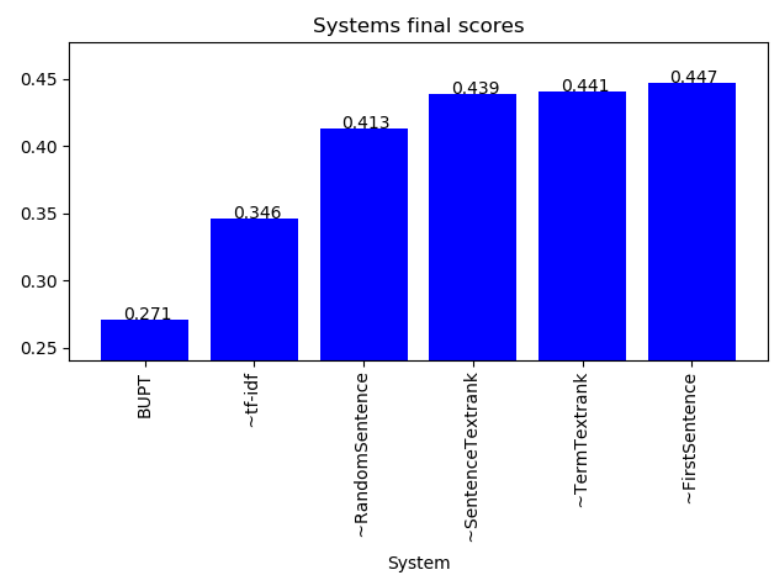

Figure 2: Average scores over all metrics for all systems

metric average scores for the first sentence taken as a headline, generated by HEvAS.

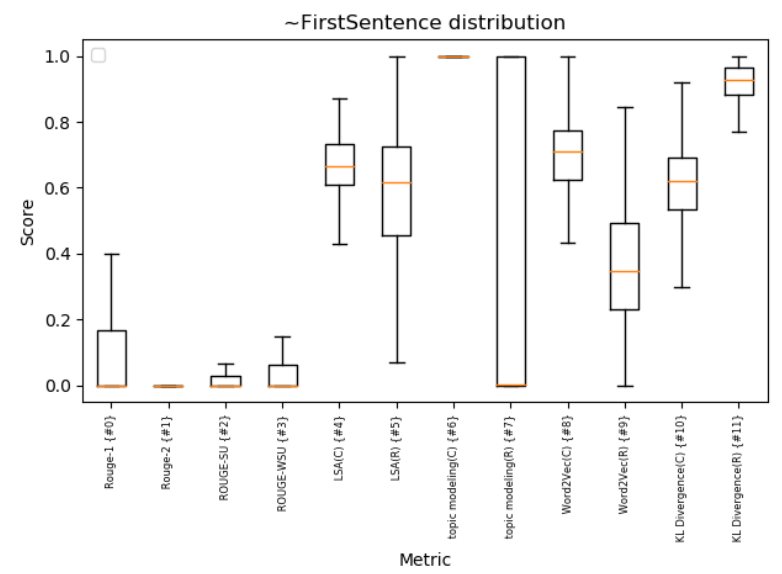

Figure 3: All average metric scores for the first sentence system

\section{Experiments}

We performed experiments on a small dataset composed of 50 wikinews articles written in English $^{7}$, where each document is accompanied by a reference (gold standard) headline. The dataset is

\footnotetext{
${ }^{7}$ Despite the experiments were performed on English documents only, some metrics of HEvAS are applicable to other languages. Extension of all HEvAS metrics to multilingual environment is a part of our future work.
} 


\begin{tabular}{|c|c|c|c|c|c|c|c|c|c|c|c|c|}
\hline System/Metric & $\mathrm{R}-1$ & R-2 & R-SU & R-WSU & LSA-C & LSA-S & TM-C & TM-S & WE-C & WE-S & KL-C & KL-S \\
\hline Random & 0.046 & 0.000 & 0.011 & 0.018 & 0.869 & 0.721 & 0.783 & 0.586 & 0.693 & 0.445 & 0.593 & 0.954 \\
\hline TF-IDF & 0.008 & 0.000 & 0.002 & 0.003 & 0.980 & 0.731 & 0.338 & 0.470 & 0.650 & 0.390 & 0.578 & 0.951 \\
\hline First & 0.408 & 0.177 & 0.176 & 0.236 & 0.892 & 0.828 & 0.925 & 0.691 & 0.734 & 0.735 & 0.664 & 0.959 \\
\hline STextRank & 0.191 & 0.066 & 0.061 & 0.096 & 0.904 & 0.781 & 0.794 & 0.608 & 0.732 & 0.579 & 0.692 & 0.932 \\
\hline WTextRank & 0.263 & 0.009 & 0.082 & 0.114 & 0.857 & 0.768 & 0.923 & 0.663 & 0.735 & 0.613 & 0.719 & 0.906 \\
\hline
\end{tabular}

Table 1: Mean scores of informativeness metrics.

\begin{tabular}{cccccc}
\hline System/Metric & Fog & NR & PNR & PR & AWL \\
\hline Random & 0.740 & 0.471 & 0.004 & 0.004 & 6.318 \\
TF-IDF & 0.786 & 0.511 & 0.000 & 0.000 & 6.853 \\
First & 0.410 & 0.396 & 0.006 & 0.017 & 5.154 \\
STextRank & 0.446 & 0.357 & 0.005 & 0.020 & 4.987 \\
WTextRank & 0.863 & 0.584 & 0.002 & 0.000 & 6.040 \\
\hline
\end{tabular}

Table 2: Mean scores of readability metrics.

publicly available. ${ }^{8}$ Table 1 contains mean scores per each informativeness metric (with default settings) for all five baselines (see Section 3.3). Each metric, except ROUGE, was applied for a coverage (denoted by $C$ suffix) and a similarity (denoted by $S$ suffix) scenarios. Table 2 contains the results of readability metrics for all baselines.

The results of a correlation analysis ${ }^{9}$ between informativeness metrics demonstrate a high correlation between all ROUGE metrics and between ROUGE metrics and Word Embedding similaritybased metric (WE-S). Figure 4 shows correlation achieved for ROUGE-1 and ROUGE-SU metrics. However, a low correlation was obtained between

Rouge-1 \{\#0\} - ROUGE-SU \{\#2\} correlation:0.999

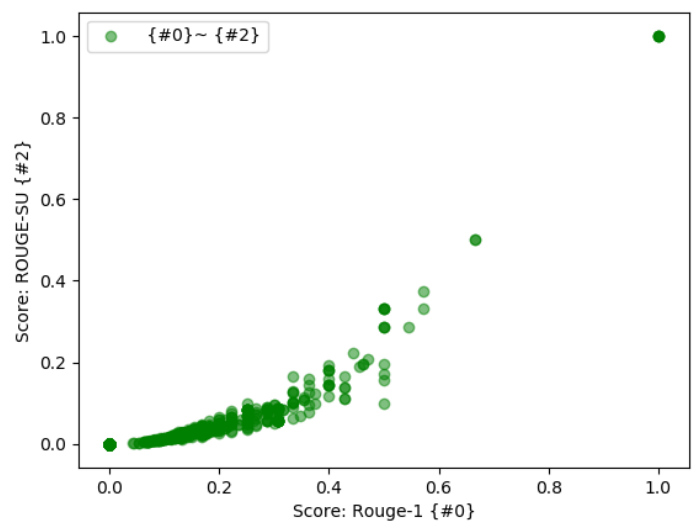

Figure 4: Correlation between ROUGE-1 and ROUGE-SU metrics

all other metrics. Also, coverage metrics usually

\footnotetext{
${ }^{8}$ https: / / drive.google.com/file/d/ 1 JHKH 4- 4 9UwbKdx 7MIXJaLZSd 44 AUKFC/view? usp=sharing

${ }^{9}$ Performed for the First baseline using Pearson correla-
}

do not correlate with the similarity metrics of the same type (an exception-correlation 0.6-was observed in a case of TM-based metrics). There are no correlated readability metrics. The lowest negative correlation $(-0.5)$ was found between AWL and Gunning Fog Index and between PNR and NR. Detailed correlation scores between different metrics achieved for our dataset are given in Table 3.

\section{Conclusions and future work}

In this paper we presented a working system named HEvAS for automated headline evaluation. The HEvAS system provides a user with 12 metrics, where some of them are novel, which measure headline quality in terms of informativeness-topics coverage and closeness to the human-generated headlines. Also, HEvAS provides five readability metrics, which measure how understandable the headlines. The system provides an output which enables to rank different systems by their scores. Most HEvAS metrics are adaptable to multiple languages. However, some metrics require an additional effort, such as training word vectors or applying a POS tagger on a corpus in a target language. Our future work includes the following tasks: (1) extension of our system with more metrics, especially metrics measuring the grammatical correctness of the generated headlines; (2) adaptation of HEvAS to multiple languages (in progress); and (3) measuring the correlation of automatic metrics with the human judgements (in progress). 


\begin{tabular}{ccccccccccccc}
\hline & R-1 & R-2 & R-SU & R-WSU & LSA-C & LSA-S & TM-C & TM-S & WE-C & WE-S & KL-C & KL-S \\
\hline R-1 & - & 0.74 & 0.99 & 0.99 & -0.30 & 0.43 & 0.24 & 0.19 & 0.07 & 0.85 & 0.34 & 0.09 \\
R-2 & 0.745 & - & 0.76 & 0.78 & -0.16 & 0.35 & 0.07 & 0.07 & -0.09 & 0.66 & 0.11 & 0.21 \\
R-SU & 0.991 & 0.76 & - & 1.00 & -0.32 & 0.41 & 0.23 & 0.18 & 0.03 & 0.84 & 0.32 & 0.12 \\
R-WSU & 0.995 & 0.78 & 1.00 & - & -0.31 & 0.42 & 0.23 & 0.18 & 0.05 & 0.85 & 0.32 & 0.11 \\
LSA-C & -0.305 & -0.16 & -0.32 & -0.31 & - & 0.28 & -0.17 & -0.11 & -0.14 & -0.21 & -0.21 & 0.01 \\
LSA-S & 0.427 & 0.35 & 0.41 & 0.42 & 0.28 & - & 0.26 & 0.24 & -0.08 & 0.44 & -0.03 & 0.05 \\
TM-C & 0.237 & 0.07 & 0.23 & 0.23 & -0.17 & 0.26 & - & 0.60 & 0.28 & 0.38 & 0.18 & -0.03 \\
TM-S & 0.187 & 0.07 & 0.18 & 0.18 & -0.11 & 0.24 & 0.60 & - & 0.11 & 0.28 & 0.06 & -0.05 \\
WE-C & 0.075 & -0.09 & 0.03 & 0.05 & -0.14 & -0.08 & 0.28 & 0.11 & - & 0.26 & 0.44 & -0.39 \\
WE-S & 0.849 & 0.66 & 0.84 & 0.85 & -0.21 & 0.44 & 0.38 & 0.28 & 0.26 & - & 0.29 & 0.00 \\
KL-C & 0.345 & 0.11 & 0.32 & 0.32 & -0.21 & -0.03 & 0.18 & 0.06 & 0.44 & 0.29 & - & -0.34 \\
KL-S & 0.088 & 0.21 & 0.12 & 0.11 & 0.01 & 0.05 & -0.03 & -0.05 & -0.39 & 0.00 & -0.34 & - \\
\hline
\end{tabular}

Table 3: Metric correlation scores.

\section{References}

Reinhard Bergmann, John Ludbrook, and Will PJM Spooren. 2000. Different outcomes of the wilcoxonmannwhitney test from different statistics packages. The American Statistician 54(1):72-77.

D M Blei. 2012. Probabilistic topic models. Соттиnications of the ACM 55(4):77-84.

D. M. Blei, A. Y. Ng, and M. I. Jordan. 2003. Latent dirichlet allocation. Journal of Machine Learning Research 3:993-1022.

Meri Coleman and Ta Lin Liau. 1975. A computer readability formula designed for machine scoring. Journal of Applied Psychology 60(2):283.

Kevyn Collins-Thompson. 2014. Computational assessment of text readability: A survey of current and future research. ITL-International Journal of Applied Linguistics 165(2):97-135.

Carlos A Colmenares, Marina Litvak, Amin Mantrach, and Fabrizio Silvestri. 2015. Heads: Headline generation as sequence prediction using an abstract feature-rich space. In Proceedings of the 2015 Conference of the NAACL: HLT. pages 133-142.

William H DuBay. 2004. The principles of readability. Online Submission.

Shawn Graham, Scott Weingart, and Ian Milligan. 2012. Getting started with topic modeling and mallet. Technical report, The Editorial Board of the Programming Historian.

Robert Gunning. 1952. The technique of clear writing.

Julia Hancke, Sowmya Vajjala, and Detmar Meurers. 2012. Readability classification for german using lexical, syntactic, and morphological features. Proceedings of COLING 2012 pages 1063-1080.

Yuko Hayashi and Hidekazu Yanagimoto. 2018. Headline generation with recurrent neural network. In New Trends in E-service and Smart Computing, Springer, pages 81-96.

Anna Huang. 2008. Similarity measures for text document clustering. In sixth New Zealand computer science research student conference (NZCSRSC2008). pages 49-56.
Lyle V Jones and John W Tukey. 2000. A sensible formulation of the significance test. Psychological methods 5(4):411.

J Peter Kincaid, Robert P Fishburne Jr, Richard L Rogers, and Brad S Chissom. 1975. Derivation of new readability formulas (automated readability index, fog count and flesch reading ease formula) for navy enlisted personnel

M. J. Kusner, Y. Sun, N. I. Kolkin, and K. Q. Weinberger. 2015. From word embeddings to document distances. In ICML.

Chin-Yew Lin. 2004. Rouge: A package for automatic evaluation of summaries. In Proceedings of the Text Summarization Branches Out workshop.

G Harry Mc Laughlin. 1969. Smog grading-a new readability formula. Journal of reading 12(8):639646.

Rada Mihalcea and Paul Tarau. 2004. Textrank: Bringing order into text. In Proceedings of the EMNLP.

Tomas Mikolov, Kai Chen, Greg Corrado, and Jeffrey Dean. 2013. Efficient estimation of word representations in vector space. arXiv preprint arXiv:1301.3781

Jeffrey Pennington, Richard Socher, and Christopher D Manning. 2014. Glove: Global vectors for word representation. In EMNLP. ACL, volume 14, pages 1532-1543.

Luz Rello, Ricardo Baeza-Yates, Laura DempereMarco, and Horacio Saggion. 2013. Frequent words improve readability and short words improve understandability for people with dyslexia. In IFIP Conference on Human-Computer Interaction. Springer, pages 203-219.

Shiqi Shen, Yu Zhao, Zhiyuan Liu, Maosong Sun, et al. 2016. Neural headline generation with sentence-wise optimization. arXiv preprint arXiv:1604.01904

Christian Smith, Henrik Danielsson, and Arne Jönsson. 2012. A good space: Lexical predictors in vector space evaluation. In LREC 2012. Citeseer, pages 2530-2535. 
Sanja Štajner, Richard Evans, Constantin Orasan, and Ruslan Mitkov. 2012. What can readability measures really tell us about text complexity. In Proceedings of workshop on natural language processing for improving textual accessibility. Citeseer, pages 14-22.

David Zajic, Bonnie Dorr, and Richard Schwartz. 2002. Automatic headline generation for newspaper stories. In Workshop on Automatic Summarization. 